\title{
Magnetization Decay in CoCr-alloy Perpendicular Magnetic Recording Media
}

\author{
Yoshiyuki HIRAYAMA, Kenya ITO, Yukio HONDA, and Masaaki FUTAMOTO \\ Central Research Laboratory, Hitachi, Ltd., Kokubunji, Tokyo 185, Japan
}

\begin{abstract}
The magnetization decay in CoCr-alloy perpendicular magnetic recording media has been investigated. Both remanent magnetization and recorded signal output decay rates of CoCrPtTa medium increase with decreasing magnetic layer thickness. The decay rates can be kept small by controlling the microstructure of magnetic layer by employing a $\mathrm{CoCr}_{35} / \mathrm{TiCr}_{10}$ dualunderlayer. $\mathrm{CoCrPt}$ media with the dual-underlayer have high $\mathrm{M}_{\mathrm{r}} / \mathrm{M}_{\mathrm{S}}$ ratio $\left(\mathrm{M}_{\mathrm{r}} / \mathrm{M}_{\mathrm{S}}>0.95\right)$ and show little decay of remanent magnetization and recorded signal output, though the magnetic layer is very thin $(25 \mathrm{~nm})$ and is composed of small grains. It is possible to prepare perpendicular media comprising a Co-based alloy with $\mathrm{M}_{\mathbf{r}} / \mathrm{M}_{\mathbf{s}}>0.95$, which is an important factor to suppress the recorded signal decay rate within an acceptable level.
\end{abstract}

Key words: perpendicular magnetic recording media, CoCr-alloy, magnetization decay, signal output decay, remanent magnetization, magnetic layer thickness, underlayer

\section{INTRODUCTION}

To increase the areal recording density to beyond 10 $\mathrm{Gb} / \mathrm{in}^{2}$, perpendicular recording is promising, because the recorded bit transitions are much more stable than those of longitudinal recording. Thermal decay characteristics of written di-bits in the longitudinal recording have been studied by using simulation [1,2] and experimental techniques [3], and are considered to limit the attainable linear recording density. In contrast, the di-bit transitions recorded at high linear densities are thermally stable in

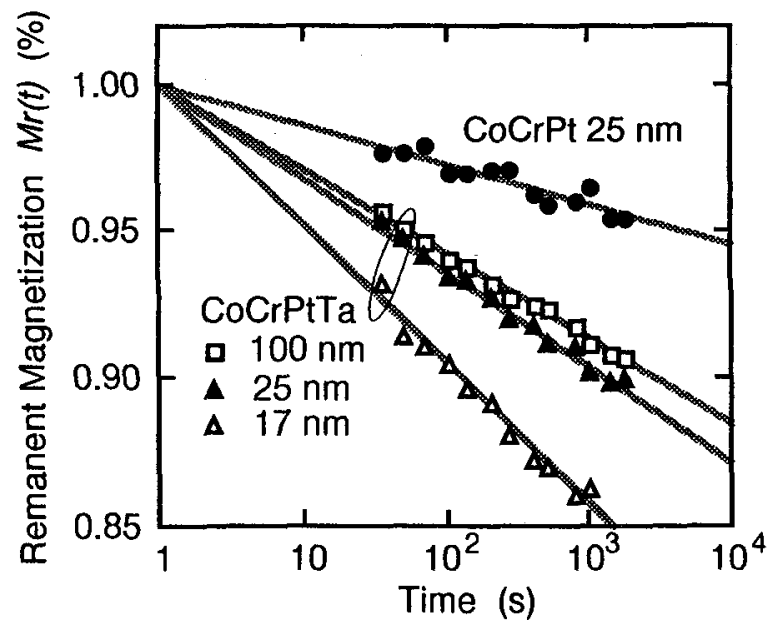

Fig. 1. Time dependences of remanent magnetization $(M r)$ measured for CoCrPtTa and $\mathrm{CoCrPt}$ perpendicular media with various magnetic layer thicknesses. perpendicular recording. However, magnetic fluctuations formed inside the recorded bits due to the demagnetizing field cause medium noise and signal output decay. Especially, the output decay becomes more serious [4,5], when the magnetic grain size is decreased to reduce the medium noise $[6,7]$. In this study, we investigate the relationships between remanent magnetization decay, recorded signal output decay and magnetic properties of $\mathrm{CoCr}$-alloy single-layer perpendicular recording media.

\section{EXPERIMENTAL PROCEDURES}

Thin film media were prepared on 3.5 inch diameter surface oxidized Si substrates by DC magnetron sputtering at substrate temperatures of $230-260^{\circ} \mathrm{C}$ using an ultra-high vacuum sputtering system [8]. $\mathrm{TiCr}_{10}$ and paramagnetic $\mathrm{CoCr}_{35}$ films were deposited as underlayers to enhance the c-axis oriented columnar growth of $\mathrm{CoCr}_{19} \mathrm{Pt}_{10}$, $\mathrm{CoCr}_{19} \mathrm{Pt}_{10} \mathrm{Ta}_{2}$ or $\mathrm{CoCr}_{17}$ Taz magnetic layers. $5 \mathrm{~nm}$ thick carbon film was deposited on the medium surface as a protective layer.

Magnetic properties were measured using a vibrating sample magnetometer (VSM). All the magnetic properties were measured by applying the magnetic field perpendicular to the film surface. Magnetization decay data were obtained by averaging more than ten series of values measured within the time range of 36 and $1800 \mathrm{~s}$. after reducing the applied magnetic field from 13 down to $0 \mathrm{kOe}$. To investigate the differences of signal output decay for the various types of media or for various linear recording densities, an inductive write head with track width $\left(\mathrm{T}_{\mathrm{w}}\right)$ of $2.7 \mu \mathrm{m}$ and gap length $\left(\mathrm{G}_{1}\right)$ of $0.4 \mu \mathrm{m}$, and an MR head $\left(T_{w}=2.5 \mu \mathrm{m}, G_{1}=0.25 \mu \mathrm{m}\right)$ were used. The sensitivity

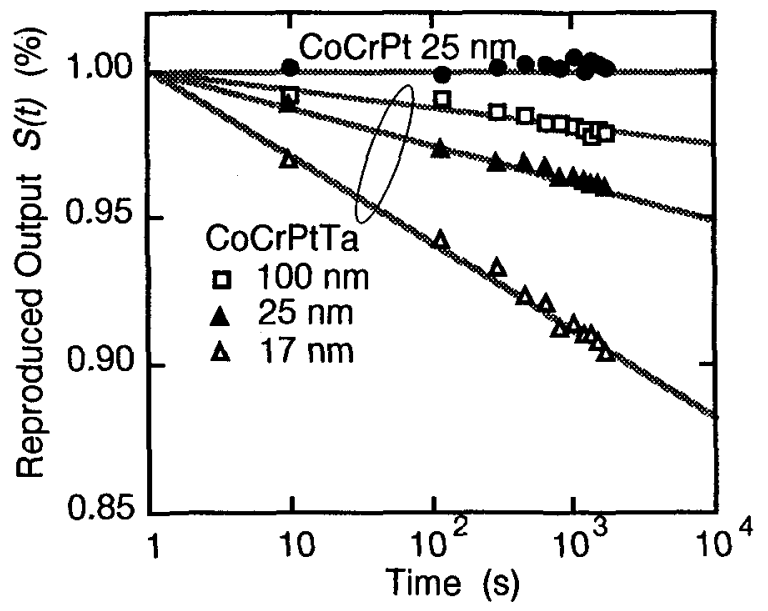

Fig. 2. Time dependences of output $(S)$ measured for 40 $\mathrm{kFCl}$ signals written in $\mathrm{CoCrPtTa}$ and $\mathrm{CoCrPt}$ perpendicular media with various magnetic layer thicknesses. 


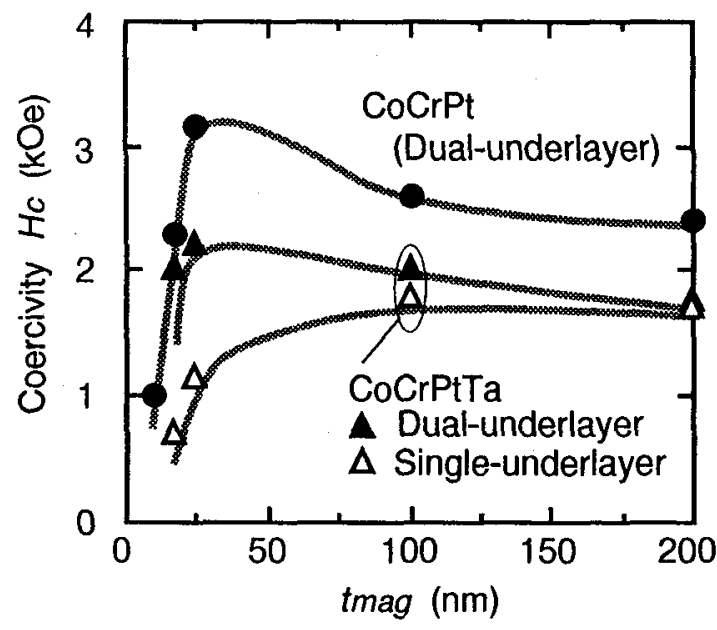

Fig. 3. Magnetic layer thickness (tmag) dependences of coercivity $(H c)$ measured for $\mathrm{CoCrPtTa}$ and $\mathrm{CoCrPt}$ perpendicular media.

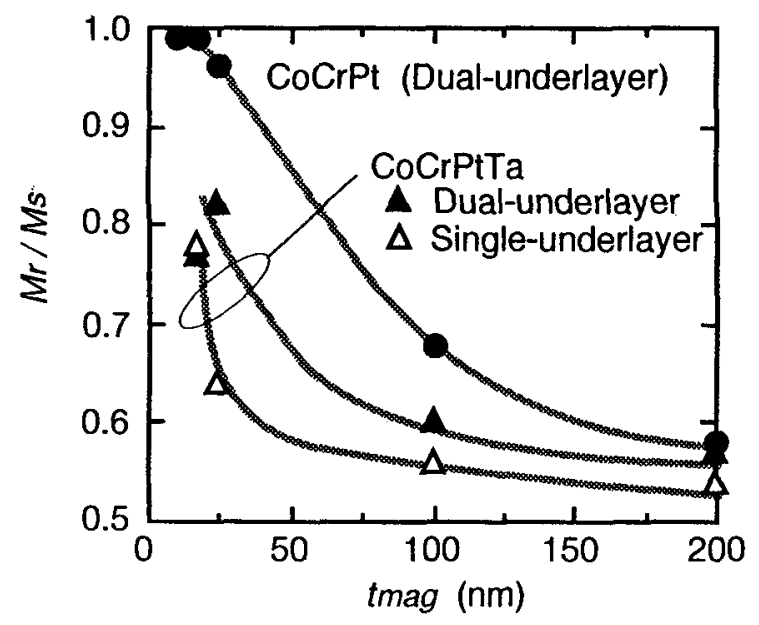

Fig. 4. Magnetic layer thickness (tmag) dependences of remanent to saturation magnetization ratio $(M r / M s)$ measured for $\mathrm{CoCrPtTa}$ and $\mathrm{CoCrPt}$ perpendicular media.

of the MR head was calibrated by referring to newly written signals in the neighboring tracks at each measurement.

\section{RESULTS AND DISCUSSION}

Time dependences of remanent magnetization $\left(\mathrm{M}_{\mathrm{r}}(\mathrm{t})\right)$ measured for the CoCrPtTa and the CoCrPt perpendicular media are shown in Fig. 1. The remanent magnetization of each medium decays in proportion to the logarithm of time. Decay rates $\left(\Delta \mathrm{M}_{\mathrm{r}}\right)$ can be estimated from the slopes of the experimental data. Figure 2 shows the time dependences of output $(S(t))$ measured for the $40 \mathrm{kFCI}$ signals written on the same media as shown in Fig. 1. The signal output decay $(\Delta S)$ is similar to the magnetization decay. However, the rates of the signal output decay are smaller than those of the magnetization decay. Therefore, the stability of recorded information for the perpendicular media can be estimated by measuring the
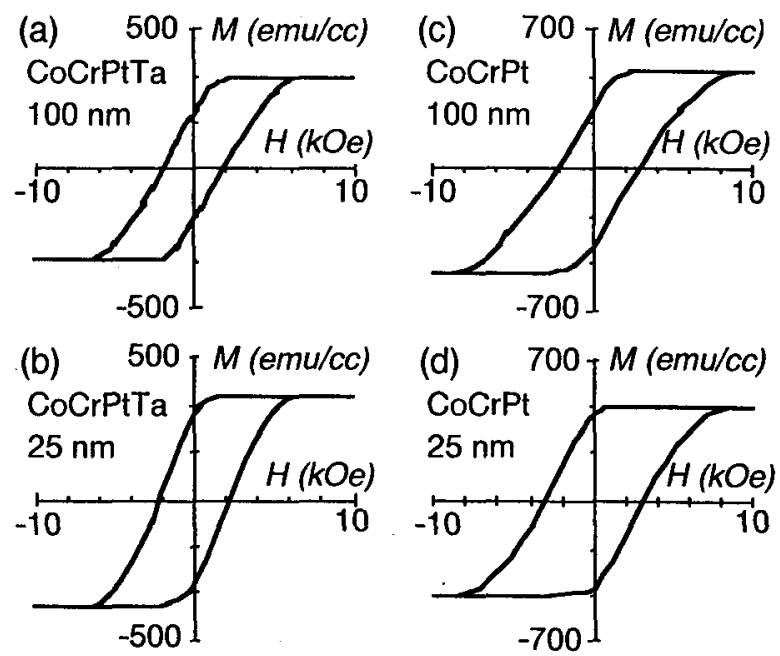

Fig. 5. Magnetization curves measured for CoCrPtTa and CoCrPt films with layer thickness of 100 or $25 \mathrm{~nm}$ formed on a $\mathrm{CoCr} 35 / \mathrm{TiCr} 10$ dual-underlayer.

remanent magnetization decay as the worst case. In the present study, the magnetic layer thickness ( $t_{\mathrm{mag}}$ ) dependences of the magnetic properties and the decay characteristics are examined for the CoCrPtTa and the CoCrPt perpendicular media to clarify what is the most effective factor in reducing the magnetization decay. Coercivity $\left(\mathrm{H}_{\mathrm{C}}\right)$, remanent to saturation magnetization ratio $\left(\mathrm{M}_{\mathrm{r}} / \mathrm{M}_{\mathrm{s}}\right)$, magnetization curves, remanent magnetization decay rates $\left(\Delta \mathrm{M}_{\mathrm{r}} / \mathrm{M}_{\mathrm{r}}(1)\right)$ and signal output decay rates $(\Delta S / S(1))$ of these media are shown in Figs. 3-7.

First, the magnetization decay behaviors of the CoCrPtTa media were investigated for the two series of the media with different underlayer structures. One is with the $\mathrm{TiCr}_{10}$ single-underlayer and the other is with the nonmagnetic $\mathrm{CoCr}_{35} / \mathrm{TiCr}_{10}$ dual-underlayer. Employment of non-magnetic $\mathrm{CoCr}$ underlayer is effective in removing the initial growth layer around the $\mathrm{CoCrPtTa} / \mathrm{TiCr}$ interface $[6,7]$. The medium noise has decreased by $40-50 \%$ by introducing the dual-underlayer when compared to medium with the $\mathrm{TiCr}$ single-underlayer. The presence of dualunderlayer also influences in the $\mathrm{H}_{c}$ (Fig. 3) and the $\mathrm{M}_{\mathbf{r}} / \mathrm{M}_{\mathbf{S}}$ (Fig. 4) characteristics. The differences are more apparent when the magnetic layer thickness is smaller than $25 \mathrm{~nm}$. Here, the influence of the initial growth layer on the magnetic properties becomes more significant by decreasing the total magnetic layer thickness as the volume fraction of the initial growth layer increases. To obtain high $H_{C}$ and high $M_{r} / M_{S}$ ratios for thin media ( $t_{\text {mag }}<25$ $\mathrm{nm}$ ), the magnetic layer should be more uniform and have no initial growth layer. The effect of the dual-underlayer is seen in the difference in magnetization decay rate shown in Fig. 6. The magnetization decay rate increases rapidly by decreasing the magnetic layer thickness below $25 \mathrm{~nm}$, and this tendency is much more remarkable in the case of the media with the $\mathrm{TiCr}$ single-underlayer. By decreasing the magnetic layer thickness, the magnetic crystal grain size and the magnetic domain size simultaneouly decrease. Small magnetic domains are considered to be thermally 


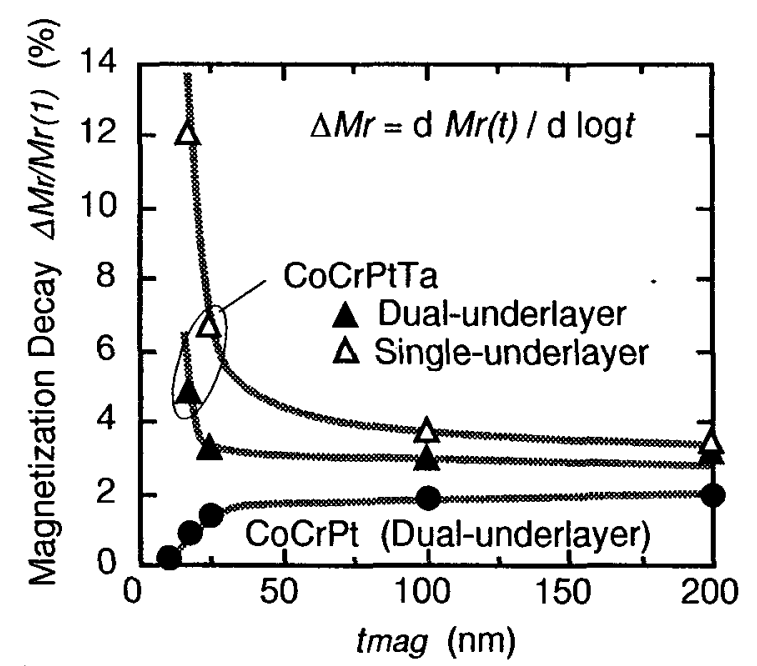

Fig. 6. Magnetic layer thickness (tmag) dependences of remanent magnetization decay rate $(\Delta M r / M r(I))$ measured for CoCrPtTa and CoCrPt perpendicular media.

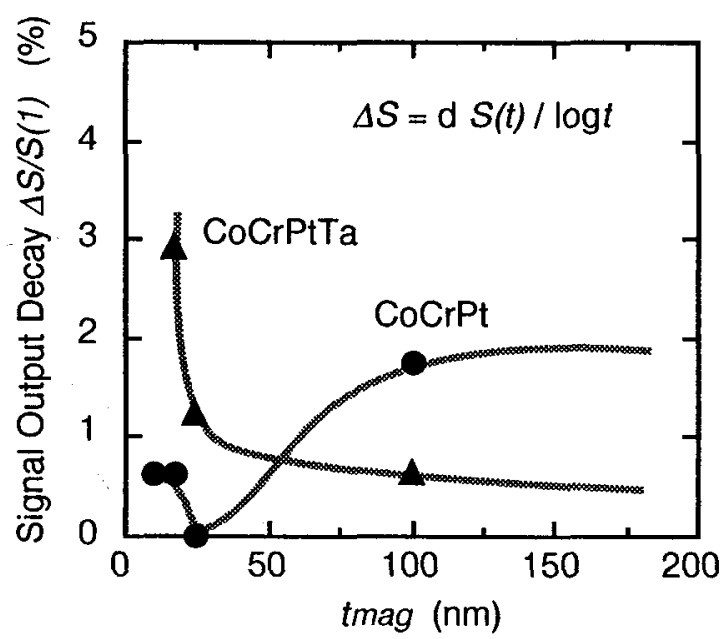

Fig. 7. Magnetic layer thickness (tmag) dependences of output decay rate $(\Delta S / S(1))$ measured for $40 \mathrm{kFCI}$ signals written in CoCrPtTa and CoCrPt media with dual-underlayer.

unstable and will reverse easily under the influence of thermal or magnetic energies. Such magnetic domain size effects seem to become more pronounced when the magnetic layer thickness is less than $25 \mathrm{~nm}$, especially in the case of the single-underlayer medium, which includes many small grains near the $\mathrm{CoCrPtTa} / \mathrm{TiCr}$ interface. It is thus important to control the grain size to keep the magnetization decay rate at a low level.

The $\mathrm{CoCrPt}$ perpendicular media show higher $\mathrm{H}_{\mathrm{c}}$ and $\mathrm{M}_{\mathrm{r}} / \mathrm{M}_{\mathrm{S}}$ values than those of the CoCrPtTa media for the magnetic layer thickness range investigated as shown in Figs. 3 and 4. The $\mathrm{M}_{\mathrm{r}} / \mathrm{M}_{\mathrm{s}}$ ratio of thin $\mathrm{CoCrPt}$ media ( tmag $<25 \mathrm{~nm}$ ) is very high and nearly equal to $1\left(\mathrm{Mr}_{\mathrm{r}} / \mathrm{M}_{\mathrm{s}}\right.$ $>0.95$ ). The magnetization curves of these media are shown in Fig. 5. Similar properties have been reported for the very thin $\mathrm{CoCr}$ films [9] and this phenomenon was explained by using the columnar microstructure model
$[10,11]$. As shown in Fig. 6, the magnetic layer thickness dependence of magnetization decay measured for the $\mathrm{CoCrPt}$ media is different from the others. The decay rates in the media with high $\mathrm{Mr}_{\mathrm{r}} / \mathrm{M}_{\mathrm{S}}$ ratio $\left(\mathrm{Mr}_{\mathrm{r}} / \mathrm{M}_{\mathrm{S}}>0.95\right)$ are less than $1.4 \%$ per decade of time though the magnetic layer thickness is smaller than $25 \mathrm{~nm}$ and the film is composed of very small grains. High $\mathrm{M}_{\mathrm{r}} / \mathrm{M}_{\mathrm{S}}$ ratio seems to have a special effect on the magnetization decay. These films are considered to have the columnar microstructures which effectively reduce demagnetizing field applied normal to the film plane. Output signal decay rates of these media are also small as shown in Fig. 7. The 25-nm-thick $\mathrm{CoCrPt}$ medium shows no decay within the measurement time range of 1 hour as shown in Fig. 2. To make the $\mathrm{M}_{\mathrm{r}} / \mathrm{M}_{\mathrm{S}}$ ratio close to 1 is an effective way of obtaining the perpendicular media with little signal decay. However, judging from the output decay data measured for 10 or 17 $\mathrm{nm}$ thick CoCrPt media shown in Fig. 7, the magnetic layer thickness of less than $17 \mathrm{~nm}$ may be too small to keep the recorded bits stable against the thermal fluctuation.

Small magnetization decays have been reported for the $\mathrm{Pd} / \mathrm{Co}$ multilayer perpendicular media [12]. As shown above, similar small decay characteristics are achievable by using the single-layer CoCr-alloy films with $\mathrm{M}_{\mathrm{r}} / \mathrm{M}_{\mathrm{S}}>$ 0.95 . But, it seems necessary to use $\mathrm{CoCr}$-alloy with high magnetic anisotropy and to control the magnetic layer thickness to obtain the properties of $M_{\mathrm{r}} / \mathrm{M}_{\mathrm{S}}>0.95$. This restriction seems to be removed when the decay rates are examined not by the magnetization decay but by the recorded signal output decay. The signal output decay rate differs depending on the linear recording density or the materials of magnetic layer, in spite of the similar magnetization decay rates. Figure 8 shows the linear recording density dependences of the signal output decay rates measured for three types of $\mathrm{CoCr}$-alloy perpendicular media. The decay rate decreases with increasing the linear recording density for all the cases and falls below $0.4 \%$ per decade of time for linear densities greater than $120 \mathrm{kFCI}$. The difference of the decay rates measured for the CoCrPt,

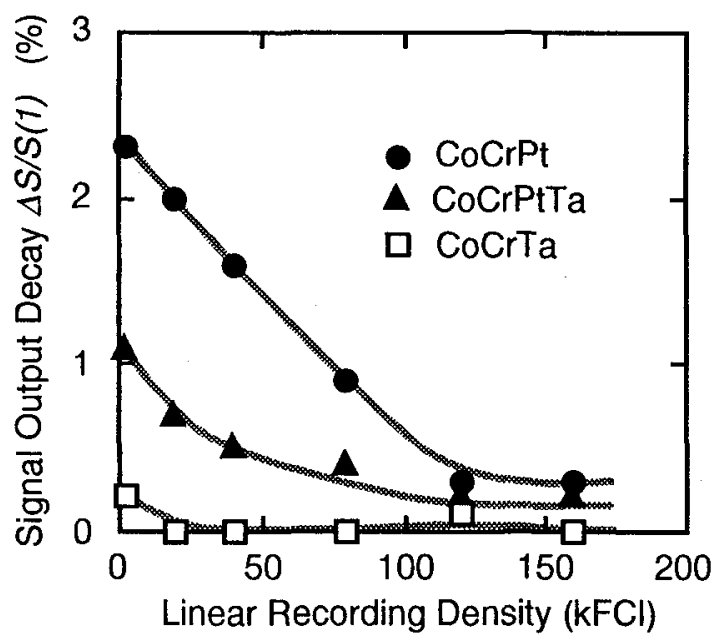

Fig. 8. Linear recording density dependences of output decay rates measured for $\mathrm{CoCrPtTa}, \mathrm{CoCrPt}$ and $\mathrm{CoCrTa}$ media with magnetic layer thickness of $100 \mathrm{~nm}$. 
the CoCrPtTa and the CoCrTa media are also clear in Fig. 8. The signal decay rates of the $\mathrm{CoCrPt}$ media measured at linear recording densities lower than $40 \mathrm{kFCI}$ are similar to that of the magnetization decay rate of $1.8 \%$ per decade time determined by using VSM. However, the signal decay rates of the CoCrPtTa and the CoCrTa are much lower than those of the respective magnetization decay rates of 2.9 and $3.3 \%$ per decade time. This difference between the signal decay rate and the magnetization decay is considered to be caused by the difference in the exchange coupling between the magnetic grains of the recording media. The CoCrTa media have the magnetically isolated microstructures with strong $\mathrm{Cr}$ segregation along the grain boundaries $[13,14]$. The weak exchange coupling between grains will probably keep the recorded bit transitions stable though the DC-erase state may not be stable. On the contrary, the $\mathrm{CoCrPt}$ media have rather strong magnetic exchange couplings between grains. The bit transitions of CoCrPt media will be more easily disturbed through the exchange force coming from the neighbor grains. Therefore, it is important to choose good materials or microstructure of the magnetic layer to obtain perpendicular media with minimal signal decay.

\section{CONCLUSIONS}

Remanent magnetization and signal output decay characteristics of single-layer perpendicular recording media have been investigated for $\mathrm{CoCr}_{19} \mathrm{Pt}_{10} \mathrm{Ta}_{2}$ and $\mathrm{CoCr}_{19} \mathrm{Pt}_{10}$ media with a $\mathrm{CoCr}_{35} / \mathrm{TiCr}_{10}$ dual-underlayer for magnetic layer thicknesses ranging from 10 and $200 \mathrm{~nm}$. The magnetization and the signal output decay rates of the CoCrPtTa media increase remarkably when the magnetic layer thickness becomes smaller than $25 \mathrm{~nm}$. The decay rates can be kept below $1.4 \%$ per decade of time by controlling the microstructure of the magnetic layer by introducing the dual-underlayer. In contrast, the magnetization decay rates of the CoCrPt media decrease with decreasing the magnetic layer thickness. The CoCrPt media with the dual-underlayer have high $\mathrm{Mr}_{\mathrm{r}} / \mathrm{M}_{\mathrm{S}}$ ratios $\left(\mathrm{M}_{\mathrm{r}} / \mathrm{M}_{\mathrm{S}}>0.95\right)$ and show little decay in both the remanent magnetization as well as the signal output, when the magnetic layer thickness is smaller than $25 \mathrm{~nm}$ where the magnetic layer is composed of small grains. Making the $\mathrm{M}_{\mathrm{r}} / \mathrm{M}_{\mathrm{S}}$ ratio close to 1 is an effective way of obtaining perpendicular media with little signal decay. Relationships between magnetization and output decay depend much on the materials used for the magnetic layer. The CoCrTa media show much smaller signal output decay characteristics than those expected from the magnetization decay behavior.

\section{ACKNOWLEDGMENTS}

We would like to thank Prof. Y. Sugita of Tohoku University for encouragement and discussions throughout the course of the research.
A part of the work was carried out under the ASET program supported by NEDO, Japan.

\section{REFERENCES}

[1] P-L. Lu and S. H. Charap, "Thermal Instability of at 10 Gbit/in² Magnetic Recording", IEEE Trans. Magn., vol. 30 , p. 4230 (1994).

[2] Y. Uesaka, Y. Nakatani and N. Hayashi, "Thermal Stability of Longitudinal and Perpendicular Recording Media Obtained by Monte Carlo Simulation", Digests of $M M M^{\prime} 96, \mathrm{~EB}-10$ (1996).

[3] Y. Hosoe, I. Tamai, K. Tanahashi, Y. Takahashi, T. Yamamoto, T. Kanbe, and Y. Yajima, "Experimental Study of Thermal Decay in High Density Magnetic Recording Media", Digests of INTERMAG'97, FC-08 (1997).

[4] T. Nagaoka, C. Baldwin, and A.P. Payne, "Magnetic and Read/Write Performance of CoCrTaPt Perpendicular Media", IEEE Trans. Magn., vol. 31, p. 2755 (1995).

[5] W. Cain, A. Payne, M. Baldwinson, and R. Hempstead, "Challenges in the Practical Implementation of Perpendicular Magnetic Recording", IEEE Trans. Magn., vol. 32, p. 97 (1996).

[6] Y. Hirayama, K. Ito, Y. Honda, and M. Futamoto, "High S/N Single-layered Perpendicular Magnetic Recording Disks", Digests of PMRS'96 , p. 95 (1996).

[7] Y. Hirayama, M. Futamoto, K. Ito, Y. Honda, and Y. Maruyama, "Development of High Resolution and Low Noise Single-layered Perpendicular Recording Media for High Density Recording", IEEE Trans. Magn., vol. 33, p. 996 (1997).

[8] Y. Matsuda, M. Suzuki, Y. Hirayama, Y. Honda, and M. Futamoto, "Co-Cr-Ta / Ti / Ti-Cr Perpendicular Recording Disks Prepared Using an UHV Sputtering System", J. Mag. Soc. Jpn., vol. 18, Suppl., p. 99 (1994).

[9] D. J. Mapps, M. A. Akhter, and G. Pan, "Very Thin CoCr Films on Titanium Underlayers for High-density Perpendicular Recording Computer Discs", IEEE Trans. Magn., vol. 26, p. 1614 (1990).

[10] T. Wielinga and J. C. Lodder, "Reversal Mechanism in Perpendicular Media with Columnar Microstructure", Phys. Stat. Sol., vol. 96, p. 255 (1986).

[11] C.-R. Chang and D. R. Fredkin, "The Shearing of Perpendicular Loop with Columnar Structure", IEEE Trans. Magn., vol. MAG-23, p. 2052 (1987).

[12] B. M. Lairson, W. Liu, and A. P. Payne, "Magnetic Relaxation at High Linear Densities in Thin Films with Perpendicular Magnetic Anisotropy", J. Appl. Phys., vol. 79, p. 7920 (1996).

[13] K. Kimoto, Y. Hirayama, and M. Futamoto, "Compositional Separation in CoCrTa Perpendicular Magnetic Thin Films", J. Magn. Magn. Mater. vol. 159, p. 401 (1996).

[14] Y. Hirayama, M. Futamoto, K. Kimoto, and K. Usami, "Compositional Microstructures of CoCr-alloy Perpendicular Magnetic Recording Media", IEEE Trans. Magn., vol. 32, p. 3807 (1996). 